\title{
Conventional CD4 + T cells regulate IL-22-producing intestinal innate lymphoid cells
}

\author{
LL Korn ${ }^{1}$, HL Thomas ${ }^{1}$, HG Hubbeling ${ }^{1,2}$, SP Spencer ${ }^{1}$, R Sinha ${ }^{3}$, HMA Simkins ${ }^{1}$, NH Salzman ${ }^{4}$, \\ FD Bushman ${ }^{3}$ and TM Laufer ${ }^{1,2}$
}

The innate and adaptive immune systems in the intestine cooperate to maintain the integrity of the intestinal barrier and to regulate the composition of the resident microbiota. However, little is known about the crosstalk between the innate and adaptive immune systems that contribute to this homeostasis. We find that CD4 + Tcells regulate the number and function of barrier-protective innate lymphoid cells (ILCs), as well as production of antimicrobial peptides (AMPs), Reg3 $\gamma$ and Reg3ß. RAG1 - / - mice lacking Tand B cells had elevated ILC numbers, interleukin-22 (IL-22) production, and AMP expression, which were corrected by replacement of CD4 + Tcells. Major histocompatibility class II $-/-(\mathrm{MHCll}-/-$ ) mice lacking CD4 + T cells also had increased ILCs, IL-22, and AMPs, suggesting that negative regulation by CD4 + T cells occurs at steady state. We utilized transfers and genetically modified mice to show that reduction of IL-22 is mediated by conventional CD4 + T cells and is T-cell receptor dependent. The IL-22-AMP axis responds to commensal bacteria; however, neither the bacterial repertoire nor the gross localization of commensal bacteria differed between $\mathrm{MHCII}+$ / - and MHCII - / - littermates. These data define a novel ability of CD4 + T cells to regulate intestinal IL-22producing ILCs and AMPs.

\section{INTRODUCTION}

The innate and adaptive immune systems collaborate at mucosal borders such as the lung, skin, and intestine to maintain barrier integrity and homeostasis with commensal microorganisms. The intestine uniquely balances requirements for nutrient breakdown and absorption with protective containment of microorganisms. Innate intestinal epithelial cells and Paneth cells, macrophages, dendritic cells, innate lymphoid cells (ILCs), and secreted mucus and antimicrobial peptides (AMPs), respond to intestinal microbes. ${ }^{1,2} \mathrm{~T}$ and $\mathrm{B}$ lymphocytes, the cells that define the adaptive immune system, contribute to intestinal homeostasis via microbial antigenspecific responses, with secretion of cytokines and bacterianeutralizing immunoglobulin A $(\operatorname{IgA}){ }^{2}$

Studies have begun to explore the cooperative interplay between innate and adaptive immunity in the intestine. For example, in the setting of defective innate functions, CD4 $+\mathrm{T}$ cells induce protective $\operatorname{IgA},{ }^{3}$ and systemic $\mathrm{B}$ cells produce bacteria-specific IgG in response to poorly contained commensals. ${ }^{4}$ Similarly, ILCs prevent systemic invasion of microbes in RAG1 - / - mice lacking adaptive immunity. ${ }^{5}$ Thus, adaptive and innate immunity compensate for each other, but whether they directly regulate each other is not well understood.

Mucosal ILCs maintain barrier homeostasis and protect against pathogens through secretion of signature cytokines. Three subclasses of ILCs in the intestine parallel the effector functions of CD4 + helper T-cell subsets: ILC1s (classical NK cells) secrete interferon gamma (IFN $\gamma$ ), ILC2s express GATA-3 and secrete interleukin-5 (IL-5) and IL-13, and ILC3s express ROR $\gamma \mathrm{t}$ and secrete IL-22 and IL-17. ${ }^{6}$ ILC3-derived IL-22 acts on IL-22 receptor-positive intestinal epithelial cells and Paneth cells to increase production of a subset of AMPs, including the Reg3 (regenerating islet-derived 3) family. Reg3 $\gamma$ and Reg3 $\beta$ neutralize Gram-positive and -negative bacteria, respectively, ${ }^{1}$ and $\operatorname{Reg} 3 \gamma$ maintains physical separation between luminal bacteria and the epithelium. ${ }^{7}$ The IL-22-AMP axis contributes

\footnotetext{
${ }^{1}$ Department of Medicine, Perelman School of Medicine at the University of Pennsylvania, Philadelphia, Pennsylvania, USA. ${ }^{2}$ Philadelphia Veterans Affairs Medical Center, Philadelphia, Pennsylvania, USA. ${ }^{3}$ Department of Microbiology, Perelman School of Medicine at the University of Pennsylvania, Philadelphia, Pennsylvania, USA and ${ }^{4}$ Department of Pediatrics, The Medical College of Wisconsin, Milwaukee, Wisconsin, USA. Correspondence: TM Laufer (tlaufer@mail.med.upenn.edu) 
to intestinal homeostasis during a variety of challenges to the intestinal barrier. ${ }^{8}$

Despite the overlap of T-cell and ILC function, adaptive immune regulation of ILCs has not been established. We used adoptive transfer and genetic approaches to demonstrate that $\mathrm{CD} 4+\mathrm{T}$ cells regulate ILC numbers, IL-22 production, and the expression of the downstream AMPs, Reg3 $\gamma$ and Reg3 $\beta$. This regulation was independent of T-cell-dependent IgA, but dependent on antigen-specific T-cell receptor (TCR) signals. The regulation by $\mathrm{CD} 4+\mathrm{T}$ cells was not mediated by changes in the intestinal microbiota as the presence or absence of $\mathrm{CD} 4+\mathrm{T}$ cells had no effect on the microbiota composition. Therefore, we have defined a novel ability of CD $4+\mathrm{T}$ cells to regulate this critical innate immune component.

\section{RESULTS}

\section{IL-22-dependent innate responses are regulated by the adaptive immune system}

To determine if the IL-22/Reg pathway is regulated by the adaptive immune system, we utilized quantitative real-time PCR to compare mRNA expression of the IL-22-responsive AMPs, Reg $3 g$ and Reg $3 b$, in total RNA from the distal small intestine, cecum, and proximal large intestine of age-matched RAG1 - / - and RAG1-heterozygous $(+/-)$ het mice. AMP mRNA levels were increased in RAG1 - / - mice on average six- or four-fold (Reg3g and Reg3b, respectively) in the small intestine, and approximately 100 - or 200-fold in the cecum and large intestine (Figure 1a). Reg protein production is regulated by both epithelial and Paneth cell-intrinsic mechanisms and in response to cell-extrinsic production of the cytokine, IL-22. Elevated AMP levels in RAG1 - / - mice were uniformly associated with approximately 25 -fold increased expression of IL-22 mRNA in the small intestine and 30- to 40-fold in the large intestine and cecum. Therefore, in the absence of adaptive immunity, the IL-22-dependent bacterial sensing pathway was enhanced.

To ask if the altered expression of IL-22 and AMPs in RAG1 - / - mice could be regulated, the adaptive immune systems of RAG1 - / - mice were reconstituted with $\sim 50 \times 10^{6}$ cells from the spleen and mesenteric lymph nodes of wild-type (WT) mice. T and B cells were reconstituted both peripherally and in the small intestine lamina propria (Si-LP) and restored small intestine luminal IgA to WT levels by 8 weeks after transfer (Figure 1 $\mathbf{b}$ and $\mathbf{c}$ ). Transfer of lymphocytes was associated with decreases in small intestine AMP and IL-22 mRNA levels of approximately 2 - and 10-fold, respectively (Figure 1a). Similar results were obtained at 3 and 6 weeks after transfer (data not depicted) and comparable decreases were observed in the cecum and large intestine. Therefore, IL-22dependent innate responses are regulated by the adaptive immune system.

ILCs are a dominant IL-22 producer in the intestine and a key upstream regulator of Reg protein production by intestinal epithelial and Paneth cells., ${ }^{2,8}$ We compared the number and function of ILCs in the small intestines of RAG1 - / - mice with those of age-matched RAG1 $/ /-$ mice. The total numbers of Si-LP and small intestine intraepithelial ILCs were increased four- to sixfold in RAG1 - / - mice compared with RAG1 + / - mice (Figure 2c). The frequency of ROR $\gamma \mathrm{t}$ positive ILC3 among total ILCs was slightly increased in RAG1 - / - mice, from approximately 75 to $85 \%$ (Figure 2d).

We next examined the proliferative capacity of ILC3s. Approximately $2-5 \%$ of ROR $\gamma \mathrm{t}+$ ILC 3 cells were proliferating in RAG1 + / - mice, as assessed by Ki-67 positivity; in RAG1 - / - mice, the frequency of proliferating ILC3's was approximately doubled (Figure $\mathbf{2 a}$ and $\mathbf{e}$ ). In addition to the increased ILC numbers and proliferation, the percentage of ILCs producing IL-22 was also elevated; $42 \%$ of Si-LP ILCs produced IL-22 in RAG1 - / - mice compared with $14 \%$ in RAG1 $+/-$ mice $(P<0.0001)$, and approximately $4 \%$ co-produced IL-22 and IL-17, compared with $1 \%$ in RAG1 + / mice $(P<0.0001$; Figure $2 \mathbf{e}$ and $\mathbf{g})$. Therefore, coordinate elevations in AMPs and IL-22 reflect increased ILC numbers, proliferation, and function.

CD4 $+\mathrm{T}$ cells are sufficient to regulate IL-22-dependent innate responses in an IFN $\gamma$-independent manner

$\mathrm{CD} 4+\mathrm{T}$ cells are mediators of intestinal homeostasis ${ }^{2}$ and we hypothesized that they would be sufficient to downregulate the IL-22/Reg pathway. In all, $10 \times 10^{6} \mathrm{CD} 4+\mathrm{T}$ cells sorted from the spleens and mesenteric lymph nodes of WT mice were transferred to RAG1 - / - mice. Cells transferred included naive, memory, and regulatory $\mathrm{T}$ cells (Tregs), but excluded NK1.1 + cells. Recipients were analyzed six to eight weeks later. $\mathrm{CD} 4+\mathrm{T}$ cells were found throughout the intestine (comprising $10-40 \%$ of cells in the Si-LP), whereas neither B cells nor luminal IgA were detected in recipient mice (Figure 2b). Therefore, outcomes reflected functions of $\mathrm{CD} 4+\mathrm{T}$ cells independent of the induction of IgA.

Six weeks after CD4 + T-cell transfer, we examined the number and function of Si-LP ILCs. Total ILC numbers in Si-LP and small intestine intra-epithelial of RAG1 - / recipients were reduced by two-thirds $(P<0.0001$ and $P<0.05$; Figure 2c). In parallel with reduced total ILC numbers, the percentage of ILC3 cells among total ILCs decreased to WT levels $(P<0.0001$; Figure 2d). Both CD $4+\mathrm{T}$ cells and ILCs rely on IL-7 for survival and express IL-7 receptor. ${ }^{6,9,10}$ Expression of IL-7r $\alpha$ on ILC3 cells, however, was not appreciably altered by the addition of $\mathrm{CD} 4+\mathrm{T}$ cells (Figure 2a and data not depicted). Still, the percentage of ILC3 cells that were Ki-67 + was reduced to WT levels $(P<0.0001$; Figure 2a and e). ILC-derived IL-22 in Si-LP was also reduced twofold, and the IL-17/IL-22 doubling producing population was restored to wild type $(P<0.0001$; Figure $2 \mathbf{f}$ and $\mathbf{g})$. Therefore, $\mathrm{CD} 4+\mathrm{T}$ cells were sufficient to reduce ILC proliferation, numbers, and IL-22 production.

A recent study demonstrated that a small percentage of ILC3s express major histocompatibility class II (MHCII), with functional consequences for $\mathrm{CD} 4+\mathrm{T}$ cells and inflammation in the Si-LP. ${ }^{11}$ The impact of CD4 + T cells on ILC3 expression of MHCII was therefore assessed. We found that approximately $10 \%$ of ILC3 cells expressed MHCII in RAG1 + / - mice 
a
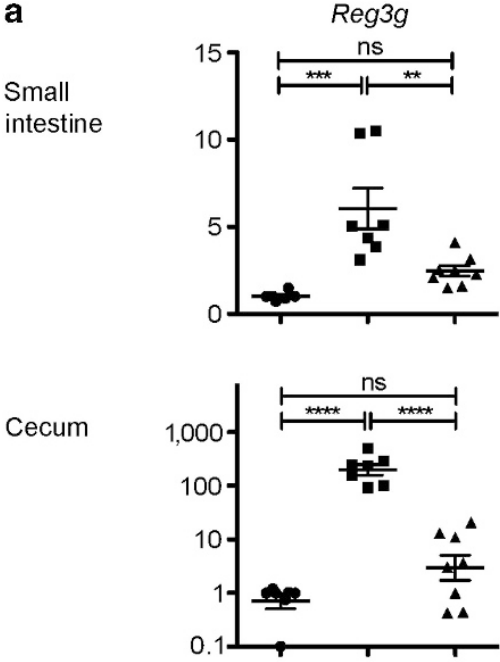

Large

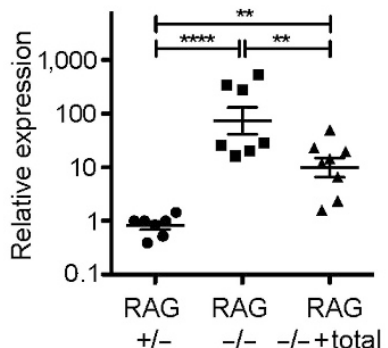

intestine

$+/-\quad-/-\quad-/-+$ total
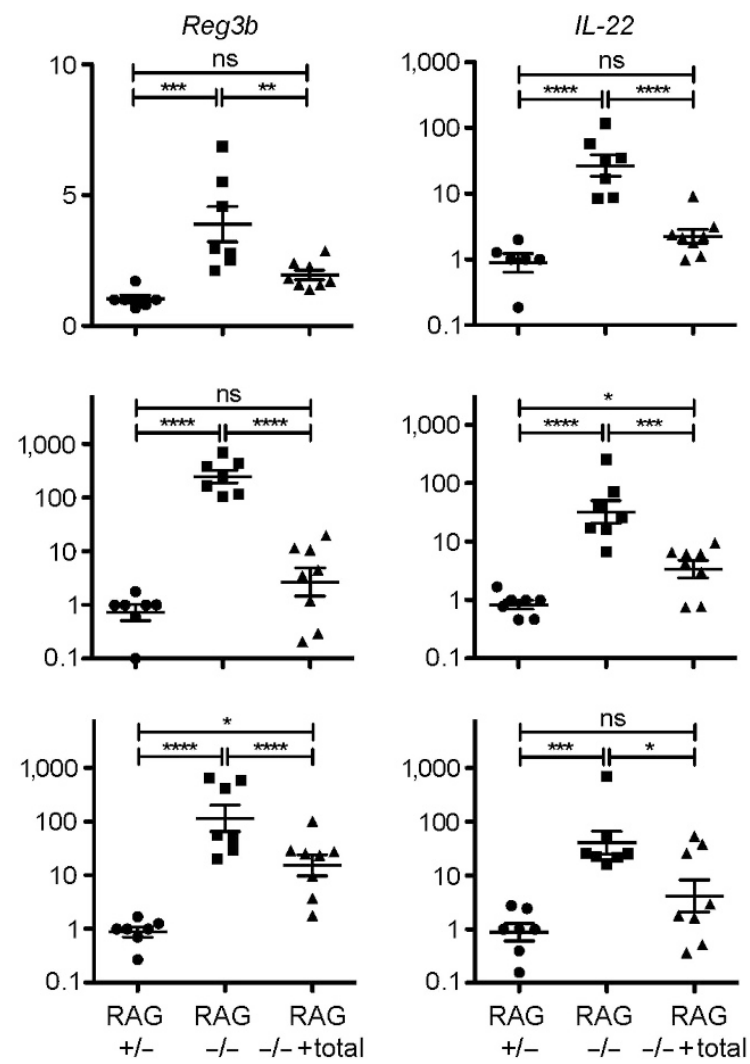

b

RAG +/-

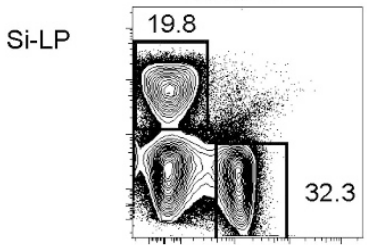

Spleen

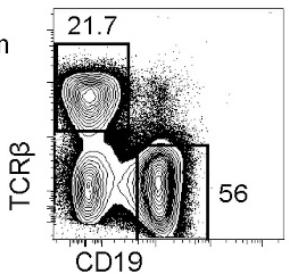

RAG-/-
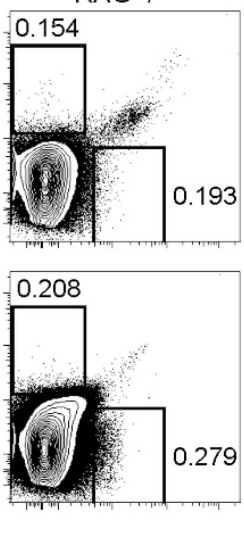
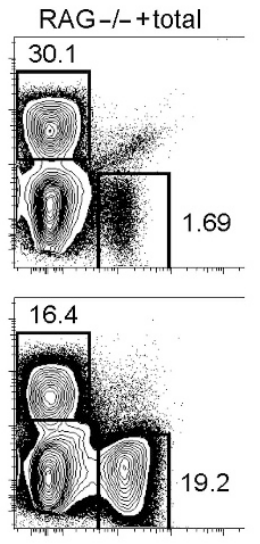

C

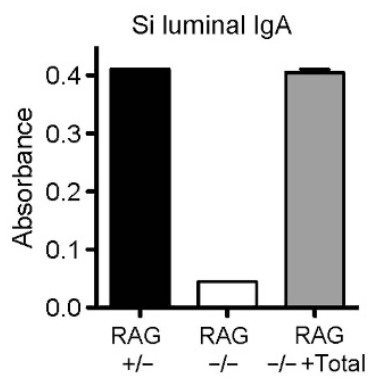

Figure 1 Interleukin-22 (IL-22)-dependent innate responses are enhanced in RAG1 - / - mice and reduced after restoration of adaptive immunity. RAG1 - / - mice were reconstituted with $50 \times 10^{6}$ cells from wild-type (WT) spleen and mesenteric lymph nodes; recipients were analyzed 8 weeks later (RAG - / - + Total). Un-manipulated RAG1 - / - and RAG1 + / - mice were controls. (a) Total mRNA from tissue sections from terminal ileum, cecum, or proximal colon was reverse transcribed and analyzed by real-time PCR. Data were analyzed using the $\Delta \Delta C T$ method normalized to a RAG1 $+/-$ for each experiment and GAPDH. Error bars show s.e.m., ${ }^{\star} P<0.05$, ${ }^{* *} P<0.01,{ }^{* * *} P<0.001,{ }^{* * * *} P<0.0001$. ns, not significant. Data pooled from three experiments. (b) Representative plots showing T cells (TCR +$)$ and B cells (CD19+) in Si-LP and spleen. (c) Bar graph of representative IgA enzymelinked immunosorbent assay small intestine luminal contents. Data in panels $\mathbf{b}$ and $\mathbf{c}$ are representative of the three experiments.

(Figure 2i and $\mathbf{j}$ ); MHCII levels on these cells were similar to those of MHCII + dendritic cells (Figure 2i). In RAG1 - / mice, only $5 \%$ of ILC 3 cells were MHCII + (Figure $2 j$ ). Six to eight weeks after the transfer of $\mathrm{CD} 4+\mathrm{T}$ cells, the percentage of ILC3 cells that was MHCII + in recipient RAG1 - / - mice was restored to WT levels (Figure $\mathbf{2 j}$ ). These data implicate $\mathrm{CD} 4+\mathrm{T}$ cells in the regulation of MHCII expression on ILC3 cells, in addition to ILC3 numbers and function.
mRNA levels of both AMPs and IL-22 were reduced in parallel with ILC numbers in RAG1 - / - recipients of CD4 + $\mathrm{T}$ cells, most strikingly in the small intestine, where AMP mRNA levels were lowered by twofold and $I L-22$ expression was lowered by threefold compared with unmanipulated RAG1 - / - mice (Figure 2h). Total intestinal IL-22 mRNA levels were decreased and the percentage of transferred CD4+ $\mathrm{T}$ cells producing IL-22 was comparable to endogenous 
production in RAG1 $+/$ - mice (Figure 3). Comparable percentages of transferred and endogenous $\mathrm{CD} 4+\mathrm{T}$ cells also generated IL-17. Fewer transferred CD4 $+\mathrm{T}$ cells produced IL-2 or IL-10, whereas significant percentage produced IFN $\gamma(P<0.0001$; Figure 3$)$. Therefore, CD4 + $\mathrm{T}$ cells are sufficient to regulate ILCs, their IL-22 production, and levels of downstream AMPs, and this regulation does not require IgA. a

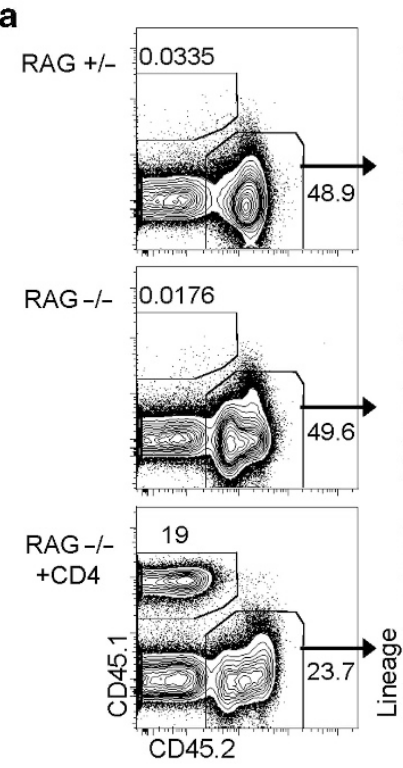

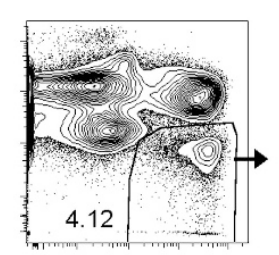
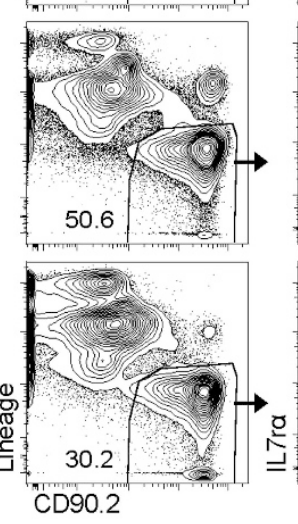

c
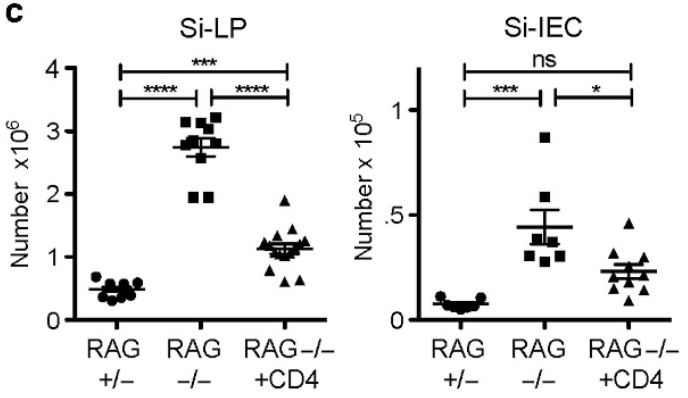

f

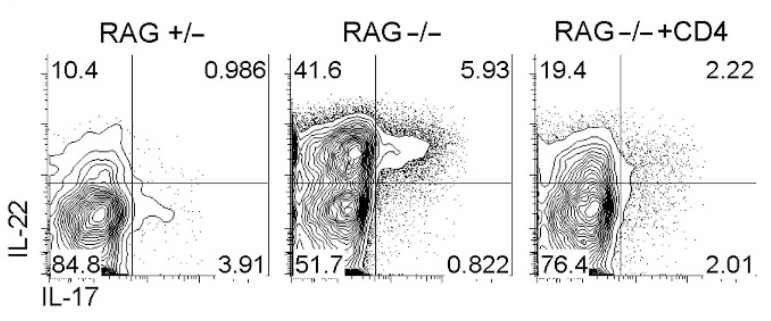

d b

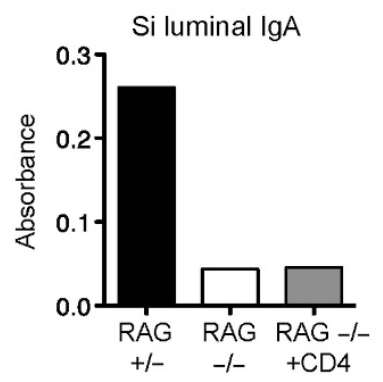

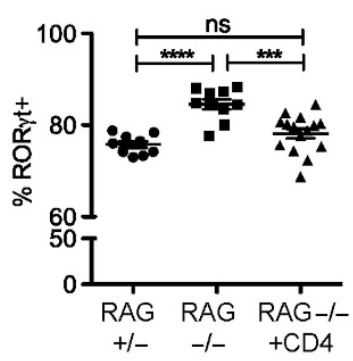

e

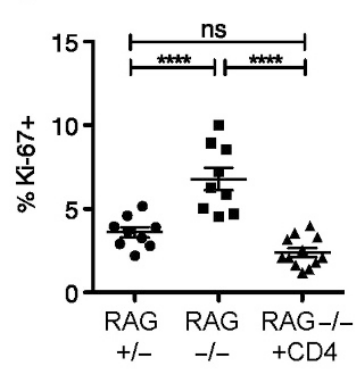

ILC cytokines
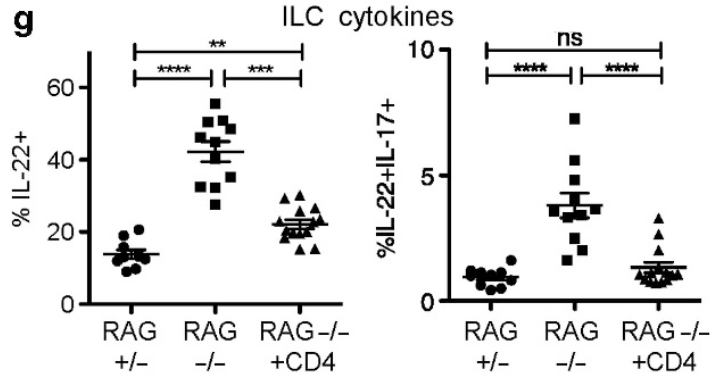

h

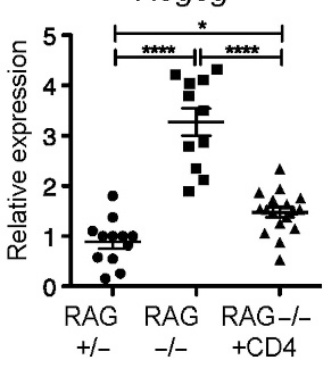

$\operatorname{Reg} 3 b$

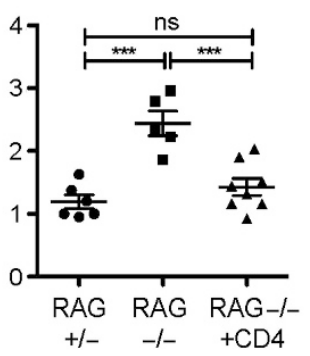

IL-22

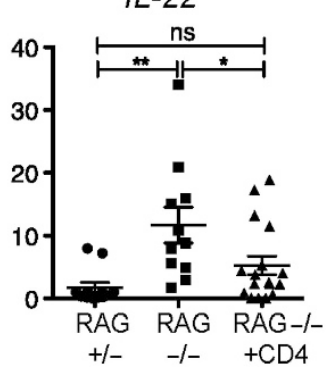

i

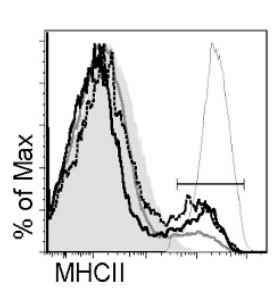

j

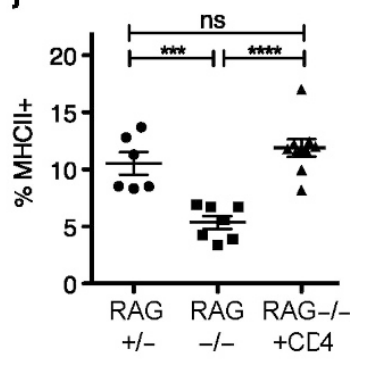


The increased IFN $\gamma$ production by $\mathrm{CD} 4+\mathrm{T}$ cells transferred to RAG1 - / - mice raised the possibility that the cytokine was regulating ILC3s; this possibility was assessed by transferring $10 \times 10^{6} \mathrm{IFN} \gamma-/-\mathrm{CD} 4+\mathrm{T}$ cells to RAG1 $-/-$ mice. Five or 6 weeks later, the transferred cells could be found in the Si-LP and, as expected, were not producing IFN $\gamma$ (Figure 4a). ILC numbers still fell by approximately two-thirds, comparable to the transfer of WT CD4 + T cells (Figure $4 \mathbf{b}$ ). The percentage of ILC3 cells that were Ki-67 + also decreased $(P<0.0001$; Figure $4 c)$. The percentages of IL-22 and IL-22/IL-17 doubleproducing ILCs were restored to WT levels $(P<0.0001$; Figure 4d). Thus, the ability of $\mathrm{CD} 4+\mathrm{T}$ cells to downregulate ILC numbers, proliferation, and function was not dependent on IFN $\gamma$. Surprisingly, RAG1 $-/-$ mice that received IFN $\gamma-/-\mathrm{CD} 4+\mathrm{T}$ cells developed wasting and grossly visible, pan-intestinal colitis (data not depicted). ${ }^{12}$
Therefore, CD4 + T cells are sufficient to regulate ILCs, their IL-22 production, and levels of downstream AMPs. This regulation does not require IgA or IFN $\gamma$ production by $\mathrm{CD} 4+$ $\mathrm{T}$ cells, and, importantly, occurs even in the presence of gross intestinal inflammation.

\section{CD4 $+\mathrm{T}$ cells are important in steady-state regulation of ILCs/AMPs}

To ask if CD4 $+\mathrm{T}$ cells regulate ILC numbers, IL-22 production, and additionally the Reg proteins at steady state, we made use of mice with more selective adaptive immune deficiencies; MHCII - / - mice lack MHCII expression and MHCII-restricted CD4 $+\mathrm{T}$ cells (Figure 5a). However, MHCII - / - and MHCII + / - mice do contain quantitatively similar amounts of IgA in the small intestine (data not depicted) in agreement with previous work showing that $\operatorname{Ig} \mathrm{A}$
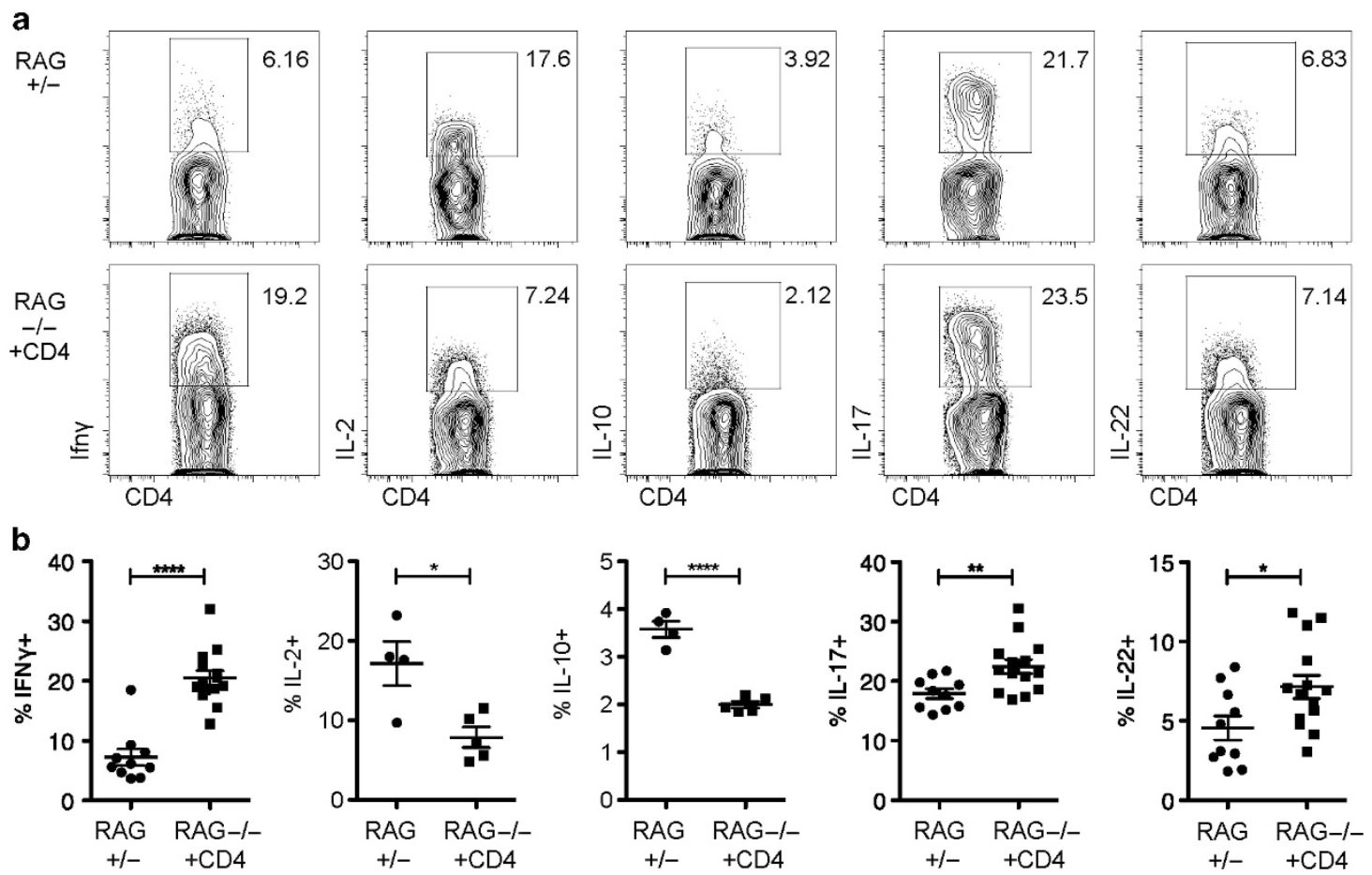

Figure 3 Transferred CD4 + T cells preferentially produce interferon gamma (IFN $\gamma$ ). Cytokine production by endogenous or transferred sorted CD4 + T cells (RAG + / - or RAG - / - + CD4, respectively) from mice analyzed 6-8 weeks after cell transfer. (a) Representative flow cytometic analysis from small intestine lamina propria (Si-LP); plots are gated on CD4 + TCR $\beta+$ cells. (b) Cytokine production by Si-LP CD $4+\mathrm{T}$ cells. Data are pooled from either two (interleukin-2 (IL-2), IL-10) or all four (IFN $\gamma$, IL-17, IL-22) of the experiments shown in Figure 2. Error bars show s.e.m., ${ }^{\star} P<0.05$, ${ }^{\star \star} P<0.01$, ${ }^{* * \star *} P<0.0001$

Figure 2 CD4 + T cells are sufficient to reduce interleukin-22 (IL-22)-dependent innate responses. RAG1 $-/-$ mice received $10 \times 10^{6}$ sorted CD4 + T cells; recipients were analyzed 6-8 weeks later (RAG - / - + CD4). (a) Representative flow cytometric analysis of transferred cells, ILCs, and Ki-67 staining on ILC3 cells in the small intestine lamina propria (Si-LP) of RAG1 + / , RAG1 - / , and RAG1 - / - CD4 mice. (b) Representative immunoglobulin $A(\operatorname{IgA})$ in the small intestinal lumen 8 weeks after transfer of CD4 + T cells. (c) Si-LP and small intestine intraepithelial (Si-IEC) innate lymphoid cell (ILC) numbers. (d) The percentage of ROR $\gamma \mathrm{t}+$, ILC3 cells among total ILCs. (e) Ki-67 + percentage among ILC3 cells. (f) Representative flow cytometric analysis of cytokine production by Si-LP ILCs; plots are gated on lineage-cd90.2 + cells. (g) Cytokine production by Si-LP ILCs. (h) Reg3g, Reg3b, and IL-22 mRNA expression in total terminal ileum (small intestine) tissue, analyzed by quantitative real-time PCR as in Figure 1. (i) Representative staining of major histocompatibility class II (MHCII) on Si-LP ILC3 cells in RAG1 - I - mice (solid light gray line), RAG1 + I - mice (dotted dark gray line), and RAG1 - I- + CD4 mice (solid black line). MHCll-negative T-cell (light gray filled) and MHCll-positive dendritic cell (thin gray line) controls from RAG1 + / - mice are also depicted. (j) Percentage of ILC3 cells that are MHCII +. Data in panels $\mathbf{c}, \mathbf{d}, \mathbf{e}, \mathbf{g}, \mathbf{a n d} \mathbf{h}(I L-22$ and Reg3g) were pooled from four experiments. Data in panels $\mathbf{h}(R e g 3 b)$ and $\mathbf{j}$ were each pooled from two of the four experiments. Error bars show s.e.m., ${ }^{\star} P<0.05$, ${ }^{\star \star} P<0.01,{ }^{\star * \star} P<0.001,{ }^{* * \star *} P<0.0001$. ns, not significant. 

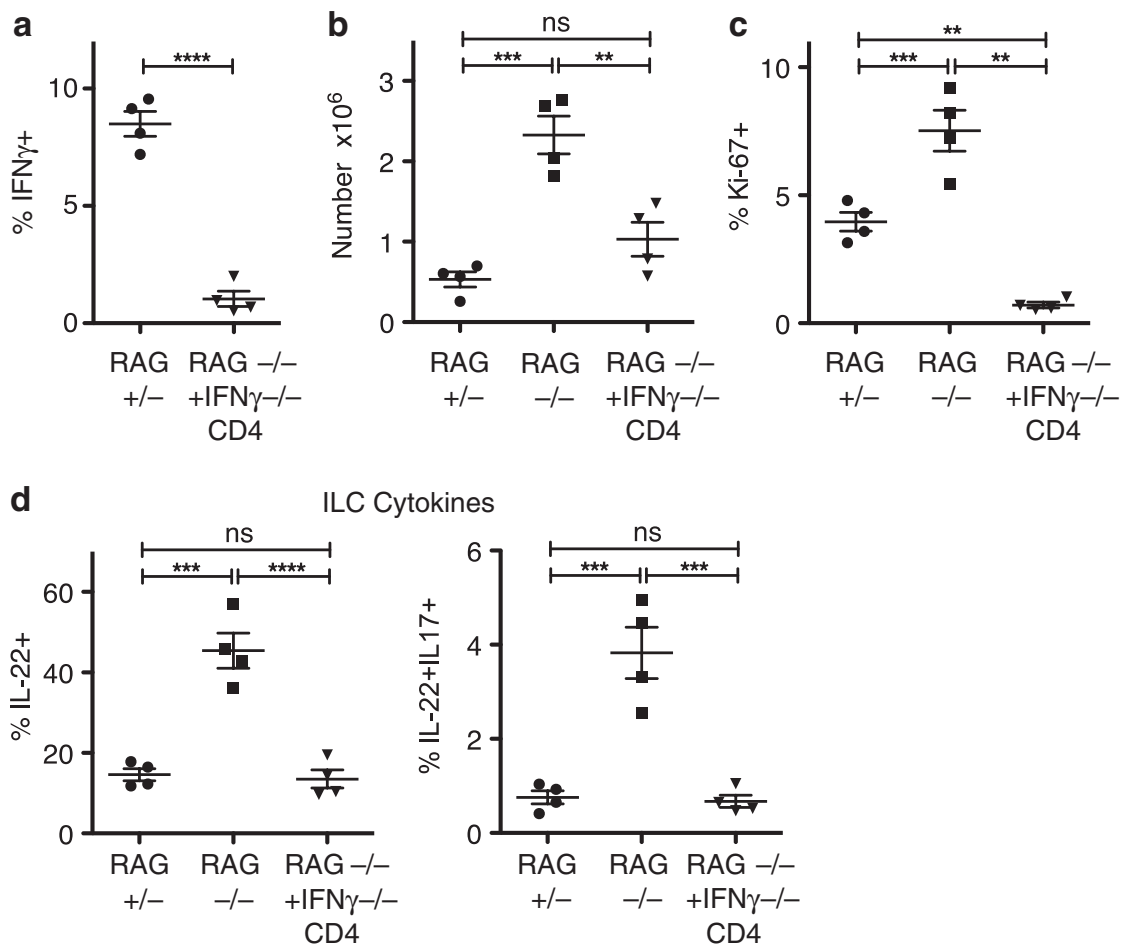

Figure 4 CD4 + T-cell regulation of innate lymphoid cells (ILCs) is independent of interferon gamma (IFN $\gamma$ ). RAG1 $-/-$ mice received $10 \times 10^{6}$ sorted IFN $\gamma-/-$ CD4 + T cells; recipients were analyzed 5 or 6 weeks later (RAG - / - + IFN $\gamma-/-$ CD4). (a) Percentage of transferred or endogenous CD4 + T cells in the small intestine lamina propria (Si-LP)-producing IFN $\gamma$. (b) Number of ILCs in the Si-LP of indicated mice. (c) Percentage of ILC3 cells that are Ki-67 + . (d) Cytokine production by Si-LP ILCs in the indicated mice. Data in panels a-d are pooled from two experiments. Error bars show s.e.m., ${ }^{\star \star} P<0.01,{ }^{\star \star \star} P<0.001,{ }^{* \star \star *} P<0.0001$. ns, not significant.

production can be T-cell independent. ${ }^{13}$ ILC numbers were increased approximately twofold in $\mathrm{MHCII}-/-$ mice compared with MHCII + / - controls, as was the percentage of ILCs producing IL-22 (Figure $\mathbf{5 b}$ and $\mathbf{c}$ ). The percentage of ILCs producing IL-22 in MHCII - / - mice was variable (7-31\%). Consistent with these observations, both $I L-22$ and Reg $3 g$ mRNA in ileum and large intestine tissue, although also variable, were also increased (Figure 5d). These data are in contrast to RAG1 - / - mice, where ILC numbers and ILCderived IL-22 and AMPs were consistently elevated.

MHCII $-/-$ mice contain CD8 + T cells, as well as NKT cells and other atypical MHCI-restricted CD4 $+\mathrm{T}$ cells. We used beta- 2 microglobulin $(\beta 2 \mathrm{~m})-/-$ mice that lack normal expression of MHCI and these T-cell populations to assess their contribution to ILC regulation. ILC numbers in $\beta 2 \mathrm{~m}-1-$ mice were intermediate between $\mathrm{MHCII}+/-/ \beta 2 \mathrm{~m}+/-$ (WT) and MHCII - / - mice although not statistically different from those in $\mathrm{MHCII} / \beta 2 \mathrm{~m}+/-$ mice, suggesting that $\beta 2 \mathrm{~m}$-restricted cells have a minimal effect on ILC numbers (Figure 5b). The percentage of ILCs producing IL-22 in $\beta 2 \mathrm{~m}-/-$ mice was equivalent to those in $\mathrm{MHCII}+/-$ animals $(12 \% \pm 0.8 \%)$, confirming that $\beta 2 \mathrm{~m}$-restricted cells are dispensable for regulation of IL-22 production by ILCs (Figure 5c). These data suggest that MHCII-restricted $\mathrm{CD} 4+\mathrm{T}$ cells (but not MHCI-restricted CD8 $+\mathrm{T}$ cells) are important to control ILC numbers, their IL-22 production, and levels of Reg $3 \gamma$. However, the variability in ILC function in
MHCII - / - mice suggests that this pathway is not absolutely required.

Would depletion of CD4 + T cells from adult WT mice have the same effect as the developmental defect in MHCII - / mice? WT mice were treated with CD4-depleting antibody (GK1.5), leading to the eradication of $\mathrm{CD} 4+\mathrm{T}$ cells from the Si-LP (Figure 6a). Six weeks after depletion, both ILC numbers and ILC3 proliferation trended upward, but differences failed to reach statistical significance (Figure $\mathbf{6 b}$ and $\mathbf{c}$ ), whereas the percentage of ILC3 cells expressing MHCII fell by one-third $(P=0.014$; Figure 6d). Similarly, the percentages of ILCs secreting IL-22 and IL-22/IL-17 trended upward; although, they were not statistically significantly increased (Figure 6e). However, the upward shifts in both ILC numbers and IL-22 production were associated with a 2.5 -fold increase in tissue mRNA expression of Reg $3 g$ in CD4-depleted mice (Figure 6f). Overall, these data are consistent with the variably increased ILC numbers, IL-22 secretion, and tissue AMP and IL-22 mRNA expression observed in MHCII - / - mice, but again suggest that $\mathrm{CD} 4+\mathrm{T}$ cells are not absolutely required in WT mice to downregulate ILC function at steady state.

\section{CD4 $+\mathrm{T}$ cells do not alter the large intestine microbiota or bacterial localization}

In our specific pathogen-free mouse facility, both IL-22 production and secondary AMP production in RAG1 - / - mice are antibiotic sensitive (Figure 7a), suggesting a dependence on 

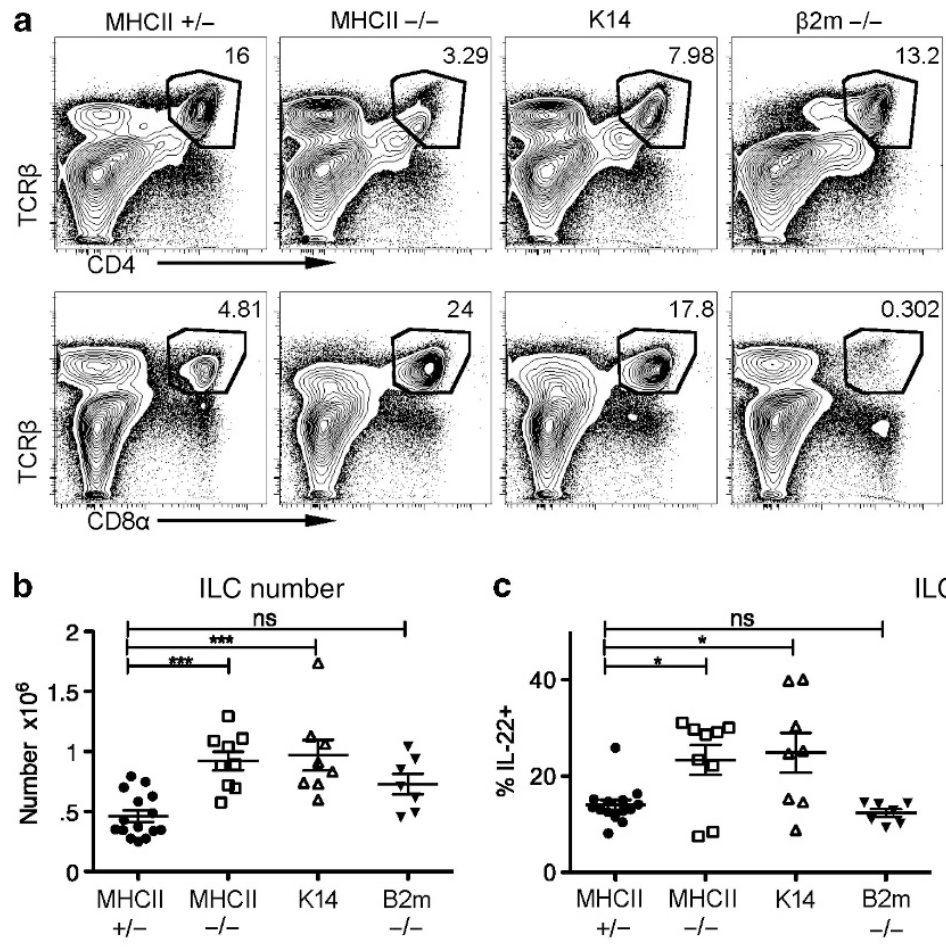

ILC cytokines
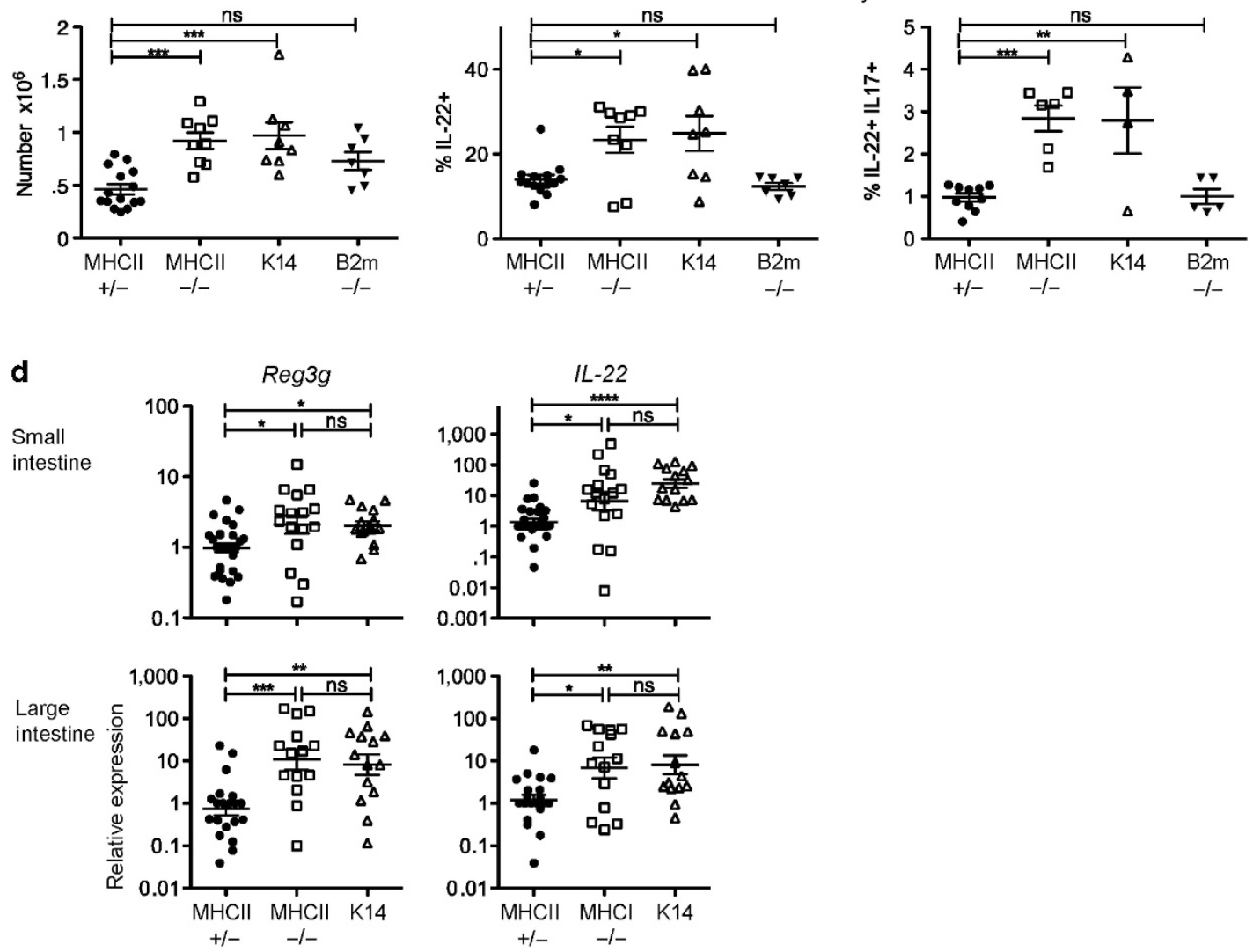

Figure 5 IL-22-dependent innate responses are enhanced in the absence of T-cell receptor (TCR)-stimulated CD $4+$ T cells. (a) Representative plots of $\mathrm{CD} 4+$ and CD8 + T cells in the small intestine lamina propria (Si-LP) of major histocompatibility class II (MHCII) $+/-, \mathrm{MHCll}-/-, \mathrm{K} 14$ (beta-2 microglobulin ( $\beta 2 \mathrm{~m}$ ) wild-type (WT) or $+/-$ ), and $\beta 2 \mathrm{~m}-/$ - (all MHCII $+/-$ ) mice. (b) Innate lymphoid cell (ILC) numbers and (c) ILC-derived cytokines in the indicated mice. (d) Reg $3 g$ and $I L-22$ mRNA expression in total intestinal tissue, analyzed by quantitative real-time PCR as in Figure 1. Error bars show s.e.m., ${ }^{\star} P<0.05,{ }^{\star *} P<0.01,{ }^{* * *} P<0.001,{ }^{* \star \star} P<0.0001$. ns, not significant. Data are pooled from six or seven experiments.

local intestinal bacteria. In antibiotic-treated RAG1 - / mice, ILC numbers additionally fell by approximately onethird, and IL-22 production decreased by twofold (Figure 7b). To address two different explanations for the enhanced response to the commensal bacterial microbiota, we asked if $\mathrm{CD} 4+\mathrm{T}$ cells altered either the repertoire of commensal bacteria and/or host sensing of the commensal bacteria.
To address differences in bacterial repertoire, we defined the composition of the commensal intestinal bacteria in MHCII - / mice and their MHCII + I - littermates. As measures in the small intestine revealed relatively few bacteria (data not depicted), we focused on the large intestine. To minimize environmental and husbandry differences that can drive microbial diversity, ${ }^{14}$ pregnant mothers were separated into 
a
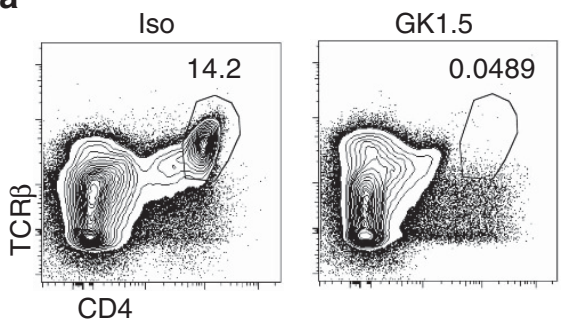

e

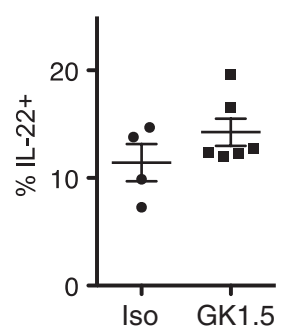

b

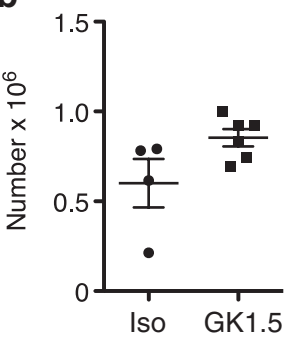

$\mathbf{f}$

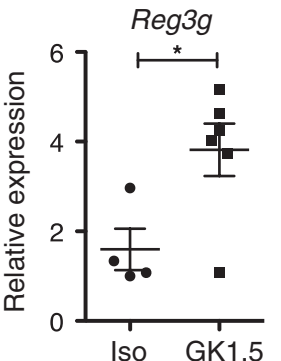

C

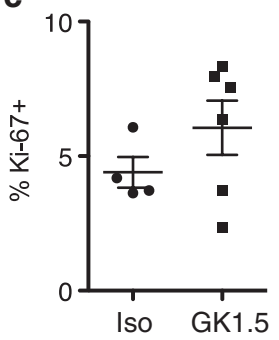

d

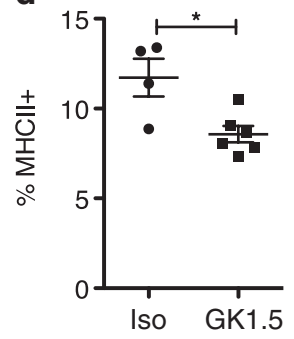

Figure 6 Depletion of CD4 + T cells in wild-type (WT) mice. Mice were treated with CD4-depleting antibody (GK1.5) or an isotype control for 6 weeks before small intestine lamina propria (Si-LP) analysis. (a) Flow cytometry of CD4 + T cells in the Si-LP of treated mice. (b-f) Graphs show (b) ILC numbers, (c) the percentage of ILC3 cells that are Ki-67 + , (d) the percentage of ILC3 cells that are MHCII +, (e) cytokine production by ILCs. (f) Reg3g, Reg3b, and IL-22 mRNA expression in total terminal ileum (small intestine) tissue, analyzed by quantitative real-time PCR. Data are pooled from two independent experiments. Error bars show s.e.m., ${ }^{*} P<0.05$.

their own cages before giving birth, and littermates were weaned into individual cages at 3-4 weeks of age and killed at 6 weeks, at which point total colonic DNA was prepared from each individual animal. Quantitative PCR analyses showed that the expression of MHCII and the presence of CD $4+\mathrm{T}$ cells had no statistically significant effect on the total numbers of either Eubacteria or major bacterial families, with the exception of a less than twofold effect on lactobacilli (Figure 7c). In addition, deep sequencing analysis of the16S ribosomal DNA genes of colonic commensals found that qualitative differences in bacterial repertoire between $\mathrm{MHCII}+/-$ and $\mathrm{MHCII}-/-$ genotypes did not achieve significance, as measured by principal coordinate analyses of litters (Figure 7d). In contrast, there were statistically significant differences in the numbers and bacterial repertoires of RAG1 - / - mice and their littermate controls (Figure $7 \mathbf{f}$ and $\mathbf{g}$ ). In the setting of these small differences, we could not detect quantitative changes in RAG1 - / - mice with reconstitution of the adaptive immune system (data not depicted). In all the strains examined, litter and maternal effects on bacterial composition dominated over genotype (Figure 7e and $\mathbf{h}$ ). These data suggest that the effects of CD4 $+\mathrm{T}$ cells on ILC biology are not secondary to altered microbial composition.

To explore the possibility of altered bacterial sensing in the absence of CD4 $+\mathrm{T}$ cells, we utilized fluorescence in situ hybridization to eubacterial $16 \mathrm{~S}$ to assess bacterial localization in the large intestine in littermate or extensively co-housed mice; differences in bacterial localization could alter bacterial sensing in the absence of compositional differences. Blinded images were scored based on the proximity of bacteria relative to epithelium (Figure 8a). Overall bacterial localization was not different in MHCII - / - mice compared with $\mathrm{MHCII}+/-$ mice, nor in RAG1 - / - mice compared to RAG1 + / - mice or CD $4+$ T-cell-reconstituted RAG1 - / - mice (Figure $\mathbf{8 b}$ and c). These data suggest that effects of CD4 $+\mathrm{T}$ cells on altered ILC biology is not driven by large-scale differences in bacterial localization.

\section{CD4 + T-cell regulation of ILCs requires MHCII-TCR interactions}

The effect of CD4 $+\mathrm{T}$ cells on IL-22-producing ILCs in the small intestine may require antigen-dependent $\mathrm{T}$-cell activation, or, alternatively, be mediated by antigen-independent competition with ILCs for a niche and survival factors. To distinguish between these possibilities, we asked if CD4+ $\mathrm{T}$ cells require antigen-specific TCR signals to regulate the IL-22/Reg pathway. We made use of K14-A $\beta^{\mathrm{b}}$ mice (K14), in which MHCII is restricted to thymic cortical epithelium; CD4 + T cells are positively selected but are not exposed to any peripheral TCR signals. ${ }^{15}$ Positive selection leads to increased frequencies of CD $4+\mathrm{T}$ cells in the Si-LP of K14 mice as compared with the residual $\mathrm{CD} 4+\mathrm{T}$-cell population in MHCII $-/-$ mice (Figure 5a). ILC numbers and the percentage of ILCs producing IL-22 in K14 mice were equivalent to those in MHCII - / - mice; both strains had increased numbers of ILCs compared with $\mathrm{MHCII}+/-$ mice (Figure 5b and c). Similarly, levels of $I L-22$ and Reg3g mRNA were increased in $\mathrm{K} 14$ and MHCII - / - mice (Figure 5d). The comparable elevations in $\mathrm{MHCII}-/-$ and $\mathrm{K} 14$ mice in ILC numbers, function, and AMP levels strongly suggest that CD4 + T cells require TCR signals to regulate ILCs, IL-22, and AMPs. 

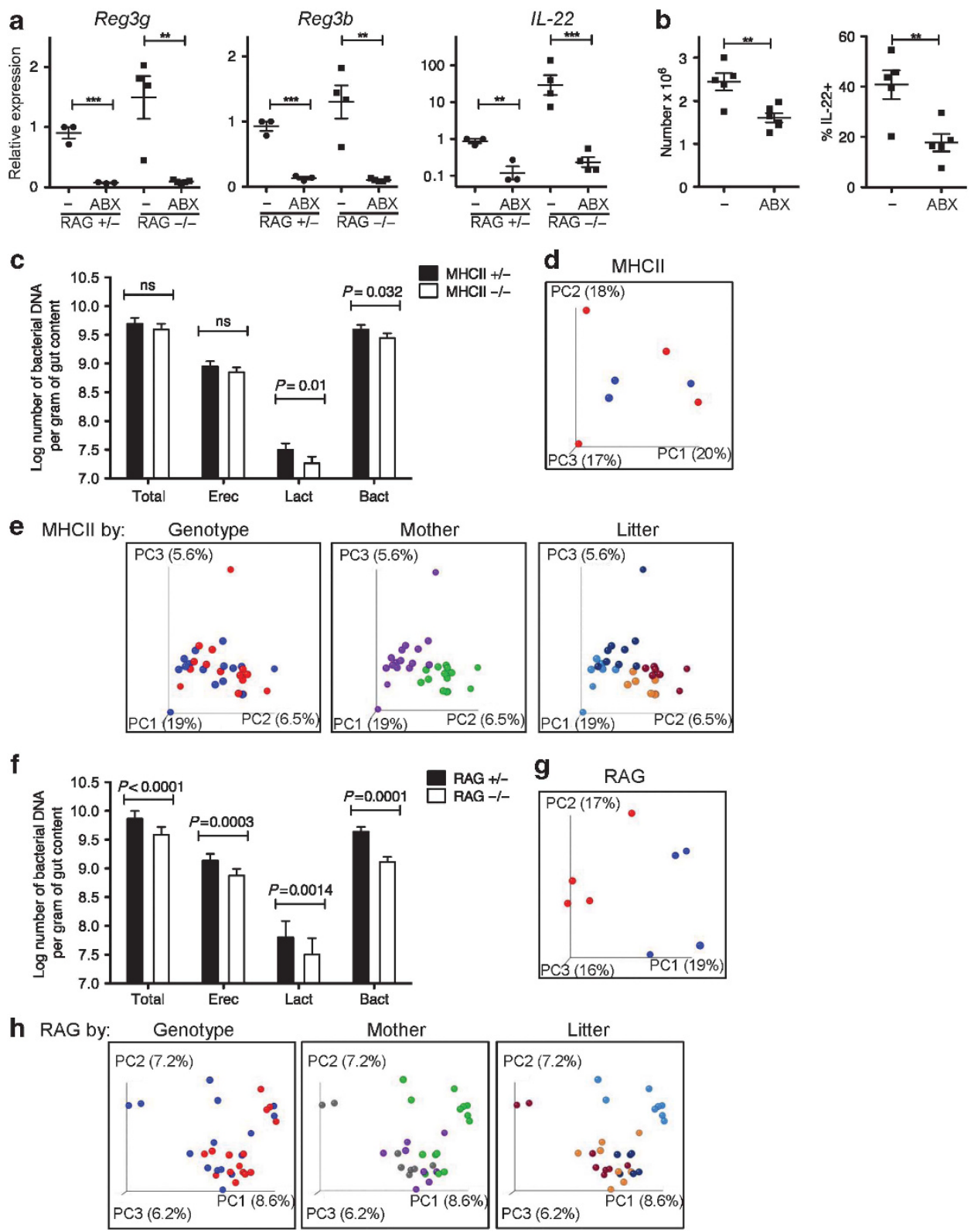

Figure 7 Impact of CD4 + T cells and adaptive immunity on the large intestine microbiota. (a) Reg3g, Reg3b, and interleukin-22 (IL-22) mRNA expression in total terminal ileum (small intestine) tissue of antibiotic-treated or control mice, analyzed by quantitative real-time PCR as in Figure 1, pooled from two experiments. (b) Innate lymphoid cell (ILC) numbers and IL-22 production in antibiotic-treated or control RAG1 - / - mice, pooled from two experiments. Error bars show s.e.m., ${ }^{* *} P<0.01,{ }^{* * *} P<0.001$. (c) Quantitative PCR of major bacterial groups in major histocompatibility class II $(\mathrm{MHCll})+/$ - and $\mathrm{MHCll}-/$ - littermates $(\mathrm{MHClI}+/-n=9-12, \mathrm{MHClI}-/-n=13-14)$, analyzed using genomic reference DNA standards. (d) Principal coordinate plot of a representative $\mathrm{MHCll}$ litter, with $+/-$ genotype shown in red and $-/-$ in blue. (e) Principal coordinate analyses of all $\mathrm{MHClI}+/$ - or $\mathrm{MHC}-/$ - mice sequenced, colored by genotype (+ I - in red and $-/$ - in blue), mother (purple and green), and litter (blue and brown tones). (f-h) The same analyses performed for MHCll mice, applied to RAG1 + / - or RAG1 - / - littermates (for e, RAG1 + / - $n=47, \mathrm{RAG} 1-/-$ $n=35)$. NS, not significant.

\section{Tregs are not sufficient to regulate ILC-derived IL-22 in the small intestine}

Foxp3 + Tregs dampen immune responses and prevent intestinal inflammation in multiple settings. ${ }^{16}$ To determine if the CD4 + effect on ILC regulation was mediated by Tregs, green fluorescent protein $+($ GFP +$)$ Tregs sorted from Foxp3-GFP reporter mice were transferred into RAG1 $-/-$ mice in numbers comparable to the number of Tregs in the initial inoculum of $10 \times 10^{6}$ total $\mathrm{CD} 4+\mathrm{T}$ cells $\left(\sim 0.5-1 \times 10^{6}\right)$. Transferred cells comprised $1-5 \%$ of cells in the Si-LP six weeks after transfer (Figure 9a). The purity of the Treg population was reduced by $40-80 \%$ after transfer, consistent with past descriptions of reconstitution of lymphopenic mice ${ }^{17}$ (Figure 9a). ILC numbers were inconsistent at six weeks after transfer (Figure 9b). However, the percentage of ILCs producing IL-22 and IL-22/IL-17, as well as levels of $I L-22$ and 


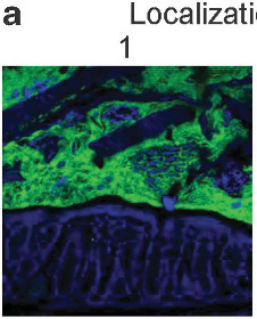

3

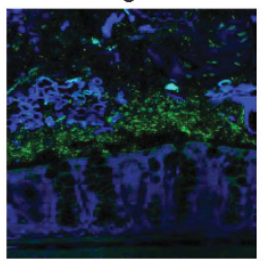

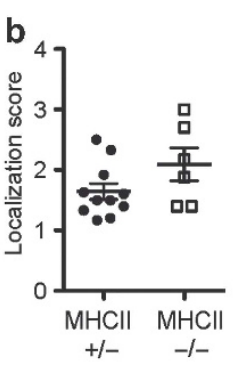

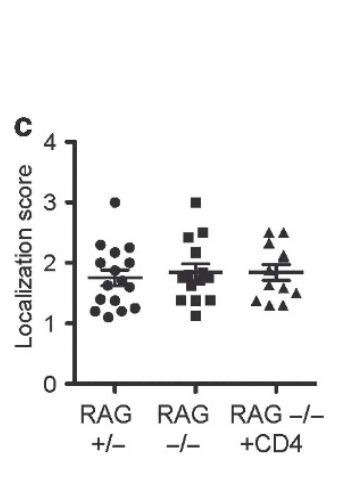

4

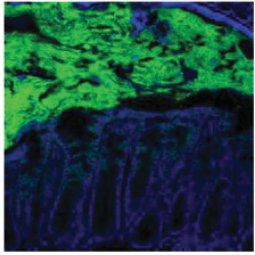

2
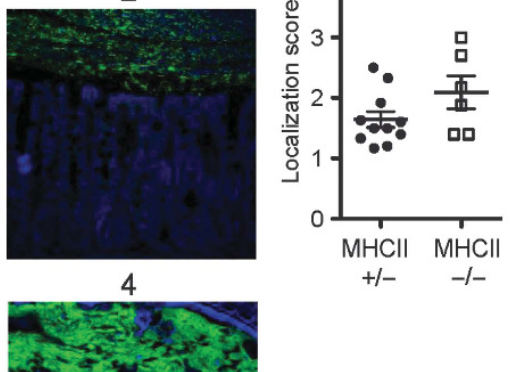

Figure 8 Large intestine bacterial localization is not altered by the presence of CD4 + T cells. (a) Representative images of fluorescence in situ hybridization (FISH) staining of large intestine demonstrating localization scores: 1 - no bacterial contact with epithelium and preserved mucus layer; 2-bacterial penetration into mucus layer without epithelial contact; 3-epithelia contact; 4-extensive epithelial contact. Images were acquired and analyzed in a blinded manner, with three to six images acquired per large intestine. Image scores were averaged to acquire a single score per mouse. (b) Localization scores from major histocompatibility class II (MHCII) + I - and MHCII - / - mice. (c) Localization scores from RAG1 + I - mice, RAG1 - / - mice, and 6-week CD4 + T-cell-reconstituted RAG1 - / - mice. Error bars show s.e.m.

a

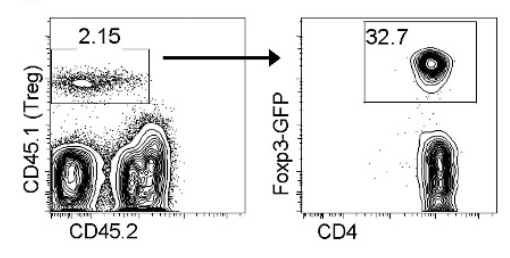

c

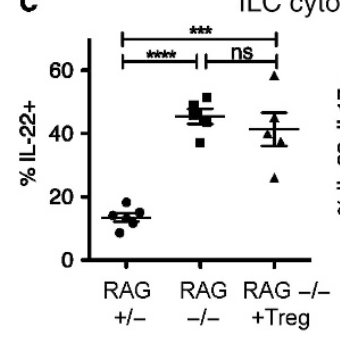

b

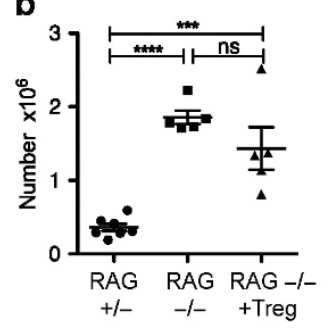

d

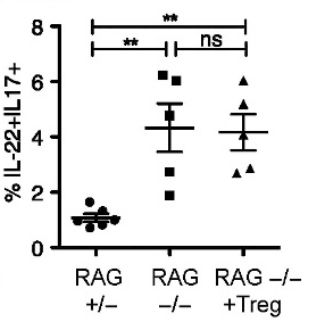

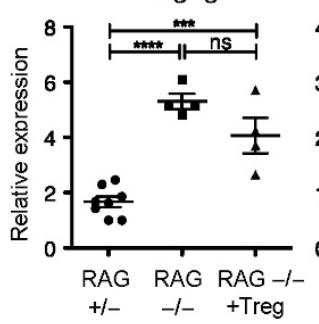

$\operatorname{Reg} 3 b$

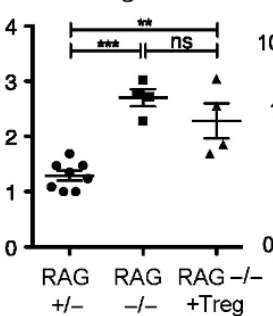

IL-22

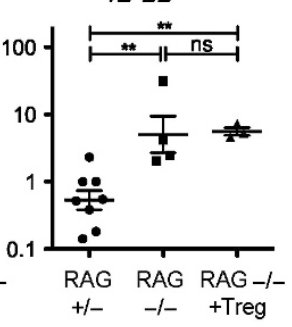

Figure 9 Tregs are not sufficient to reduce interleukin-22 (IL-22)-dependent innate responses. RAG1 $-/-$ mice that received $0.5-1 \times 10^{6}$ Tregs were analyzed 6 weeks after transfer. (a) Representative plots from small intestine lamina propria (Si-LP) showing regulatory T cell (Treg) purity. (b) Si-LP innate lymphoid cell (ILC) numbers and (c) ILC-derived cytokines from the same experiments shown in panels $\mathbf{b}$, $\mathbf{c}$ pooled from two experiments. (d) Reg3g, Reg3b, and IL-22 mRNA expression in total terminal ileum (small intestine) tissue, analyzed by quantitative real-time PCR pooled from two experiments, analyzed as in Figure 1. Error bars show s.e.m., ${ }^{\star \star} P<0.01,{ }^{\star \star *} P<0.001,{ }^{\star \star \star \star} P<0.0001$. ns, not significant.

AMP mRNA remained elevated in the small intestine (Figure 9c and d). Thus, although Tregs may influence the numbers of Si-LP ILCs, Tregs do not mediate the negative regulation exerted on ILC function by CD $4+\mathrm{T}$ cells in the small intestine.

\section{DISCUSSION}

Past studies have elucidated mechanisms by which the microbiota and the innate immune system regulate $\mathrm{CD} 4+$ T-cell responses. ${ }^{2}$ Our studies establish that conventional
$\mathrm{CD} 4+\mathrm{T}$ cells negatively regulate innate responses. We found that conventional $\mathrm{CD} 4+\mathrm{T}$ cells reduce ILC numbers, proliferation, and IL-22 production in the intestine, as well as expression of the IL-22-responsive AMPs, Reg3 $\gamma$ and Reg3 $\beta$. This regulation required neither IgA nor changes in the intestinal commensal microbiota, but required antigen-specific TCR signals. CD4 + T cells and ILCs have functional overlap; however, $\mathrm{CD} 4+\mathrm{T}$ cells and ILCs do not simply have redundant functions, as transferred $\mathrm{CD} 4+\mathrm{T}$ cells did not produce significant amounts of IL-22 and the total level of IL-22 
mRNA in CD4 + T-cell-reconstituted RAG1 $-/-$ mice was significantly decreased.

We propose that CD4 + T-cell regulation of ILCs is TCR dependent, as demonstrated by elevated ILC numbers in function in K14 mice. The TCR signals could differentiate or expand naive populations, or activate pre-existing memory populations that subsequently regulate ILCs. Although AMP expression correlated with ILC production of IL-22, separate regulatory mechanisms may also operate. A recent study showed that ILC3s express MHCII, and that this expression regulates intestinal homeostasis ${ }^{11}$ and we observed differential expression of MHCII on ILC3 cells of RAG1 $+/-$ and RAG1 - / - mice. Furthermore, CD4 + T cells were sufficient to restore levels of MHCII on ILC3 cells in RAG1 - / - to WT levels. Hepworth et al. suggested that MHCII on ILC3 cells affected CD4 + T-cell function. Our data implicate CD4+ T cells in the converse regulation of MHCII on ILC3s; although, they do not address whether that regulation is via cognate CD4 + T-cell-ILC3 interactions. Nonetheless, the putative requirement for TCR signals on CD4 + T cells to regulate ILC3 cells makes this direct interaction an attractive potential mechanism.

The ability of CD4 + T cells to regulate ILCs and AMPs may have resided within Tregs, which prevent colitis in RAG1 - / mice reconstituted with effector CD $4+\mathrm{T}$ cells. ${ }^{16}$ However, Tregs did not reduce IL-22 or AMPs in RAG1 - / - mice, while variably altering ILC numbers. Perhaps this variability stemmed from outgrowth of conventional CD4 $+\mathrm{T}$ cells from the original transferred population. Alternatively, Tregs and ILCs both utilize IL-2 and TLR2 signals, ${ }^{16,18}$ raising the possibility of signaling crosstalk between these populations. Transfer of a larger number of Tregs may have further altered ILCs. Nonetheless, these data suggest that Tregs do not have a significant role in the regulation of ILC3s.

$\mathrm{CD} 4+\mathrm{T}$ cells transferred to RAG1 $-/-$ mice produced more IFN $\gamma$ than any other cytokine; yet, WT mice contain relatively few IFN $\gamma$-producing Th1 cells in the Si-LP at steady state. Indeed, we found that CD4 $+\mathrm{T}$-cell-mediated regulation of ILCs was IFN $\gamma$ independent. Consistent with past work, ${ }^{12}$ RAG1 - / - recipients of IFN $\gamma-/-$ CD $4+$ T cells developed colitis and wasting; these data show that regulation of ILCs by $\mathrm{CD} 4+\mathrm{T}$ cells still occurs in overt inflammation. A recent study suggested that ILC3 cells may downregulate Th17 cells. ${ }^{19}$ However, the impact of IL-17 on ILCs is uncertain and future studies should directly address its role. Conversely, epithelial cell-derived IL-25 may downregulate ILC3-derived IL-22; $;^{20}$ the potential for CD4 + T-cell action in this pathway could also be explored.

Our results do not rule out that the possibility that CD4+ $\mathrm{T}$ cells and ILCs additionally compete for an anatomic niche or for a soluble factor. Although IL-7r $\alpha$ levels on ILC3 cells were not appreciably altered by the presence of CD $4+$ T cells, IL-7 might still regulate such a niche; both ILC and CD4 + T-cell survival depend on IL-7 and in the presence of exogenous IL-7, ILCs expand. ${ }^{6,9,10}$ In RAG1 - / - mice, IL-22 mRNA levels are common gamma chain dependent. ${ }^{21}$ However, it is equally likely that CD4 $+\mathrm{T}$ cells could compete with ILCs for as yet undefined metabolites.

As ILCs and AMP-producing epithelial cells respond to commensal and pathogenic bacteria and are sensitive to changes in intestinal microorganisms, $\mathrm{CD} 4+\mathrm{T}$ cells could affect ILCs and AMPs secondarily to regulating either microbial composition or sensing. In contrast to previous studies examining the microbial consequences of alterations in subsets of $\mathrm{CD} 4+\mathrm{T}$ cells, ${ }^{22,23}$ we found no significant evidence that $\mathrm{CD} 4+\mathrm{T}$ cells alter the composition of the intestinal microbiota. Eliminating specific CD4 + T-cell subsets could induce greater immune malfunction and secondary microbial dysbiosis than loss of all CD4 $+\mathrm{T}$ cells. However, in agreement with Ubeda et al., maternal and litter effects dominated over genotype differences in our hands. ${ }^{14}$ We similarly did not find altered bacterial localization that could lead to altered bacterial sensing at the epithelium. However, these studies lacked the sensitivity to reliably identify bacteria invading across the epithelium, which could also contribute to altered sensing. Future studies should also examine the small intestine, which contains fewer bacteria but where Reg $3 \gamma$ has the greatest role in bacterial localization ${ }^{7}$ and where our observed ILC effects were most robust. These data suggest that $\mathrm{CD} 4+\mathrm{T}$-cell regulation of ILCs and AMPs occurs independently of gross changes in the composition or localization of the commensal microbiota.

Along with others, we find that the microbiota has a stimulatory effect on IL-22 and AMPs. ${ }^{21,24-26}$ In contrast, a recent report by Sawa et al. found an inhibitory role for commensal microbiota on IL-22-producing ILCs except in extreme inflammation. Consistent with our data, Sawa et al. ${ }^{20}$ observed increased IL-22 production by ILCs in RAG1 - / mice, although surprisingly this did not translate into increased levels of AMPs. The regulation of ILC homeostasis and function by commensals remains unclear. ${ }^{27}$ Although confusing, these distinct data support the idea that regulatory pathways that determine IL-22 production by ILCs are both dependent and independent of the commensal microbiota; regulation by $\mathrm{CD} 4+\mathrm{T}$ cells could be a microbiota-independent pathway.

Although CD4 + T cells decreased ILC numbers and IL-22 production independent of IgA, our results do not negate a possible complementary role for this or other mucosal actors, such as $\gamma \delta$ T cells, that control bacteria at the mucosal surface or prevent intestinal injury. ${ }^{13,28}$ Indeed, in contrast to RAG1 - / mice, MHCII - / - mice had quite variable levels of ILCderived IL-22, and depletion of CD4 + T cells in WT mice did not statistically significantly alter ILCs, although $R e g 3 g$ was increased. One explanation could lie in the ability of other cell subsets to influence the signals delivered to ILCs independently of CD4 + T cells. Why this influence is variable is unclear. It is possible that ILCs respond to a specific microbiota species with differing levels across MHCII - / - mice; sequencing analyses may not be sufficiently robust to detect such differences.

We can begin to speculate how CD4 + T-cell-mediated regulation of ILCs will fit into the regulatory networks governing innate- and adaptive-immune cooperation to maintain homeostasis at steady state; whereas, most studies to date have 
examined pathologic settings. ${ }^{29-31}$ This work demonstrates a non-redundant role for conventional $\mathrm{CD} 4+\mathrm{T}$ cells in the regulation of IL-22-producing ILC3s in the intestine. Perhaps this regulation checks the potential for inappropriate innatedriven inflammation, substituting a more tailored adaptive response. Further study of this pathway should help explain the maintenance of intestinal homeostasis and could reveal additional therapeutic targets for treatment of its breakdown in disease.

\section{METHODS}

Mice. WT CD45.1, RAG1, C57BL/6J were originally purchased from Jackson Laboratories (Bar Harbor, ME). $\beta 2 \mathrm{~m} / \mathrm{A} \beta^{\mathrm{b}}-1-$ mice $^{32}$ were acquired from Taconic (Hudson, NY). MHCII - / - $\left(\mathrm{A} \beta^{\mathrm{b}}\right),{ }^{33} \mathrm{~K} 14-$ $\mathrm{A} \beta^{\mathrm{b}},{ }^{15}$ and Foxp3-GFP mice ${ }^{34}$ were bred in-house. All mice were housed under specific pathogen-free conditions (norovirus and Helicobactercolonized) in accordance with the University of Pennsylvania animal care and use guidelines, and fed autoclaved Labdiet Rodent Diet 1050 (Animal Specialties and Provisions). Mice used in ILC and transfer experiments were $2-5$ months old and were littermates or extensively co-housed. Breeding and husbandry of mice used for quantitative bacterial PCR and sequencing analyses are in Results section.

Antibodies, flow cytometry, and cell sorting. Antibodies were purchased from eBioscience (San Diego, CA), Biolegend (San Diego, CA), BD (Franklin Lakes, NJ), or Invitrogen (Grand Island, NY). Live/ Dead Fixable Aqua (Invitrogen) or 4,6-diamidino-2-phenylindole (DAPI) was used for live/dead discrimination. ILCs were defined as CD45.2 +, CD90.2 +, lineage negative. Lineage markers included TCR $\beta, C D 3, C D 11 c$, and B220 or CD19, Gr-1, CD11b, and CD5. The Foxp3/Transcription Factor Staining Buffer Set (eBioscience) was used for transcription factor stains. For intracellular cytokine stains, samples were fixed with $1.6 \%$ paraformaldehyde and stained in $0.5 \%$ saponin after stimulation. Samples were collected on a BD LSRII or FACs Canto and analyzed using Flowjo software (Treestar, Ashland, OR). For cell sorting, CD4 + T cells were enriched by negative selection as previously described, ${ }^{35}$ and further sorted on DAPI- CD4 + B220-CD8 $\beta-\mathrm{IA}^{\mathrm{b}}-\mathrm{CD} 11 \mathrm{c}-\mathrm{CD} 11 \mathrm{~b}-\mathrm{Gr} 1-\mathrm{NK} 1.1$ - cells. For Treg transfers, CD4-enriched cells from Foxp3-GFP mice were additionally sorted on GFP + cells. Sorting was performed on a BD Aria.

CD4 depletion. Mice were injected intraperitoneally with $1 \mathrm{mg}$ GK1.5 (BioXcell, West Lebanon, NH) or Rat IgG isotype control (Sigma, St Louis, MO) every 2 weeks for 6 weeks.

Antibiotic treatment. Drinking water was supplemented with ampicillin $\left(0.5 \mathrm{mg} \mathrm{ml}^{-1}\right)$, gentamicin $\left(0.5 \mathrm{mg} \mathrm{ml}^{-1}\right)$, metronidazole $\left(0.5 \mathrm{mg} \mathrm{ml}^{-1}\right)$, neomycin $\left(0.5 \mathrm{mg} \mathrm{ml}^{-1}\right)$, and vancomycin $\left(0.25 \mathrm{mg} \mathrm{ml}^{-1}\right)$ plus sucralose sweetener for 2 weeks as previously described. ${ }^{36}$

Preparation of lamina propria and intraepithelial cells. Single-cell suspensions of lamina propria were prepared by standard techniques, utilizing epithelial stripping in $1 \mathrm{~mm}$ DTT and $5 \mathrm{~mm}$ EDTA followed by digestion with $0.1 \mathrm{mg} \mathrm{m}^{-1}$ Liberase TL and Dnase (Roche, Indianapolis, IN). Intraepithelial cells were obtained from the stripped epithelial fraction after centrifugation in 30\% Percoll (GE Healthcare Life Sciences, Pittsburgh, PA). In experiments with MHCII - / $\beta 2 \mathrm{~m}-/-$, or K14 mice, Peyer's patches were first removed. Cytokine production was assessed after stimulation of LP cells with phorbol 12 -myristate 13 -acetate $50 \mathrm{ng} \mathrm{ml}^{-1}$ and Ionomycin $500 \mathrm{ng} \mathrm{ml}^{-1}$ in the presence of Brefeldin A $1 \mu \mathrm{g} \mathrm{ml}^{-1}$, at $1 \times 10^{6} \mathrm{cells} \mathrm{ml}^{-1}$ for $3.5 \mathrm{~h}$ at $37^{\circ} \mathrm{C}$.

RNA isolation and reverse transcription. Tissue pieces were cleared of stool, placed in RNAlater (Ambion, Grand Island, NY) for storage, and homogenized using a PowerGen700 Homogenizer (Fisher,
Waltham, MA). RNA isolations and reverse transcription were carried out using the RNeasy kit (Qiagen, Valencia, CA) and the High Capacity cDNA Reverse Transcription Kit (Applied Biosystems, Grand Island, NY), respectively.

Bacterial DNA isolation. Large intestines were homogenized in sterile PBS. Bacterial DNA isolation was performed using the QIAmp DNA Stool Kit (Qiagen) with optional high temperature step. ${ }^{37}$

Quantitative and real-time PCR. The following primers were utilized: ${ }^{37}$ total Eubacteria (Total), and group-specific primers for Bacteroides (Bact), Lactobacilli/enterococcus (Lact), and E. rectale/C. coccoides (Erec). Additional primer sequences were: $\operatorname{Reg} 3 g$ F: $5^{\prime}$-TTCCTGTCCTCCATGATCAAAA- ${ }^{\prime}, \operatorname{Reg} 3 g$ R: $5^{\prime}$-CATCCACC TCTGTTGGGTTCA-3'; Reg $3 b$ F: $5^{\prime}$-TCCCAGGCTTATGGCTCC TA-3', Reg3b R: 5'-GCAGGCCAGTTCTGCATCA-3'; IL-22 F: 5'-TCCGAGGAGTCAGTGCTAAA-3', IL-22 R: $5^{\prime}$-AGAACGTCTT CCAGGGTGAA-3'; GAPDH F: 5' -TCATCAACGGGAAGCCCATC AC-3', GAPDH R: 5'-AGACTCCACGACATACTCAGCACCG-3'. SYBR-green (Applied Biosystems) was utilized for all reactions.

IgA enzyme-linked immunosorbent assay. IgA enzyme-linked immunosorbent assays were performed similarly to published techniques. ${ }^{38}$ Briefly, plates were coated with 1:500 goat anti-mouse $\operatorname{Ig}(\mathrm{H}+\mathrm{L})$ (Southern Biotech, Birmingham, AL), blocked with $10 \%$ soymilk (8th Continent, Santa Ana, CA) plus 0.05\% Tween 20, incubated with small intestine lumen supernatants, followed by 1:2000 goat anti-mouse $\operatorname{Ig} \mathrm{A}(\alpha)$ detection antibody conjugated to horseradish peroxidase. Plates were developed with o-Phenylenediamine and read at $405 \mathrm{~nm}$.

454 Sequencing. Sequence determination was carried out using the 454/Roche pyrosequencing method. ${ }^{39}$ DNA samples were amplified using bar-coded DNA primers that annealed to the 16S ribosomal RNA gene within the V1V2 region essentially as described. ${ }^{40}$ Sequence reads were analyzed with QIIME pipeline ${ }^{41}$ using UniFrac. ${ }^{42,43}$ Sequence reads have been deposited in the NCBI Sequence Read Archive and are available under the SRA Project accession SRP021545.

Fluorescence in situ hybridization. 16S fluorescence in situ hybridization was performed as previously described. ${ }^{44}$ Briefly, $5 \mu \mathrm{m}$ longitudinal sections were hybridized to 16 s ribosomal RNA eubacterial probe ([AminoC6 + Alexa488]GCTGCCTCCCGTAGGAGT [AmC7-Q + Alexa488], (Eurofins MWG Operon, Huntsville, AL) at $1 \mu \mathrm{M}$. Images were acquired on a Zeiss LSM 710 confocal microscope and analyzed using Fiji (ImageJ) software. ${ }^{45}$

Statistics. Significance was determined by one-way analysis of variance with a Bonferroni post-test (Figures 1, 2, 4, and 7) or unpaired Student's $T$-test (Figures $\mathbf{3}$ and $\mathbf{5 a}$ ). Graphs shown on a log scale were log-transformed before analysis. For quantitative bacterial PCRs, a separate analysis of covariance was performed for each dependent measure (log10 bacterial count), with genotype as the fixed independent variable and parent and litter as random effects. This allowed comparison between the $+/-$ and $-/-$ genotype, while controlling for litter and parent effects. Analyses were performed using SAS software (Cary, NC).

\section{ACKNOWLEDGMENTS}

This work was supported by a pilot grant from the Penn-CHOP Joint Center for Digestive, Liver and Pancreatic Medicine P30-DK050306, the Penn Genome Frontiers Institute, and the University of Pennsylvania Center for AIDS Research (CFAR) P30 Al 045008, and R01-Al57757 (NHS). LLK was supported by the Training Program in Rheumatic Disease 5T32AR007442-25.

\section{DISCLOSURE}

The authors declared no conflict of interest.

c) 2014 Society for Mucosal Immunology 


\section{REFERENCES}

1. Gallo, R.L. \& Hooper, L.V. Epithelial antimicrobial defence of the skin and intestine. Nat. Rev. Immunol. 12, 503-516 (2012).

2. Hooper, L.V. \& Macpherson, A.J. Immune adaptations that maintain homeostasis with the intestinal microbiota. Nat. Rev. Immunol. 10, 159-169 (2010).

3. Khounlotham, M. et al. Compromised intestinal epithelial barrier induces adaptive immune compensation that protects from colitis. Immunity 37 563-573 (2012).

4. Slack, E. et al. Innate and adaptive immunity cooperate flexibly to maintain host-microbiota mutualism. Science 325, 617-620 (2009).

5. Sonnenberg, G.F. et al. Innate lymphoid cells promote anatomical containment of lymphoid-resident commensal bacteria. Science 336 , 1321-1325 (2012).

6. Spits, H. et al. Innate lymphoid cells-a proposal for uniform nomenclature. Nat. Rev. Immunol. 13, 145-149 (2013).

7. Vaishnava, S. et al. The antibacterial lectin Reglllgamma promotes the spatial segregation of microbiota and host in the intestine. Science 334, 255-258 (2011).

8. Sonnenberg, G.F., Fouser, L.A. \& Artis, D. Border patrol: regulation of immunity, inflammation and tissue homeostasis at barrier surfaces by IL-22. Nat. Immunol. 12, 383-390 (2011).

9. Schmutz, S. et al. Cutting edge: IL-7 regulates the peripheral pool of adult ROR gamma + Iymphoid tissue inducer cells. J. Immuno/ 183, 2217-2221 (2009).

10. Sprent, J. \& Surh, C.D. Normal Tcell homeostasis: the conversion of naive cells into memory-phenotype cells. Nat. Immunol. 12, 478-484 (2011).

11. Hepworth, M.R. et al. Innate lymphoid cells regulate CD4 + T-cell responses to intestinal commensal bacteria. Nature 498, 113-117 (2013).

12. Yoshida, M. et al. Differential localization of colitogenic Th1 and Th2 cells monospecific to a microflora-associated antigen in mice. Gastroenterology 123, 1949-1961 (2002).

13. Cerutti, A. \& Rescigno, M. The biology of intestinal immunoglobulin A responses. Immunity 28, 740-750 (2008).

14. Ubeda, C. et al. Familial transmission rather than defective innate immunity shapes the distinct intestinal microbiota of TLR-deficient mice. J. Exp. Med. 209, 1445-1456 (2012).

15. Laufer, T.M., Dekoning, J., Markowitz, J.S., Lo, D. \& Glimcher, L.H. Unopposed positive selection and autoreactivity in mice expressing class II MHC only on thymic cortex. Nature 383, 81-85 (1996).

16. Nutsch, K.M. \& Hsieh, C.-S. T cell tolerance and immunity to commensal bacteria. Curr. Opin. Immunol. 24, 385-391 (2012).

17. Komatsu, N. et al. Heterogeneity of natural Foxp3 + T cells: a committed regulatory $\mathrm{T}$-cell lineage and an uncommitted minor population retaining plasticity. Proc. Natl. Acad. Sci. USA 106, 1903-1908 (2009).

18. Crellin, N.K. et al. Regulation of cytokine secretion in human CD127(+) LTi-like innate lymphoid cells by Toll-like receptor 2. Immunity 33, 752-764 (2010).

19. Qiu, J. et al. Group 3 innate lymphoid cells inhibit T-cell-mediated intestinal inflammation through aryl hydrocarbon receptor signaling and regulation of microflora. Immunity 39, 386-399 (2013).

20. Sawa, S. et al. ROR $\gamma \mathrm{t}+$ innate lymphoid cells regulate intestinal homeostasis by integrating negative signals from the symbiotic microbiota. Nat. Immunol. 12, 320-326 (2011).

21. Satoh-Takayama, N. et al. Microbial flora drives interleukin 22 production in intestinal NKp46 + cells that provide innate mucosal immune defense. Immunity 29, 958-970 (2008).

22. Kawamoto, S. et al. The inhibitory receptor $\mathrm{PD}-1$ regulates IgA selection and bacterial composition in the gut. Science 336, 485-489 (2012).
23. Josefowicz, S.Z. et al. Extrathymically generated regulatory T cells control mucosal TH2 inflammation. Nature 482, 395-399 (2012).

24. Keilbaugh, S.A. et al. Activation of Reglll / and interferon expression in the intestinal tract of SCID mice: an innate response to bacterial colonisation of the gut. Gut 54, 623-629 (2005).

25. Brandl, K. et al. Vancomycin-resistant enterococci exploit antibioticinduced innate immune deficits. Nature 455, 804-807 (2008).

26. Sanos, S.L. et al. RORgammat and commensal microflora are required for the differentiation of mucosal interleukin 22-producing NKp46 + cells. Nat. Immunol. 10, 83-91 (2009).

27. Tait Wojno, E.D. \& Artis, D. Innate lymphoid cells: balancing immunity, inflammation, and tissue repair in the intestine. Cell Host Microbe 12, 445-457 (2012).

28. Ismail, A.S. et al. Gammadelta intraepithelial lymphocytes are essential mediators of host-microbial homeostasis at the intestinal mucosal surface. Proc. Natl. Acad. Sci. USA 108, 8743-8748 (2011).

29. Basu, R. et al. Th22 cells are an important source of IL-22 for host protection against enteropathogenic bacteria. Immunity 37, 1061-1075 (2012).

30. Zenewicz, L.A. et al. Innate and adaptive interleukin-22 protects mice from inflammatory bowel disease. Immunity 29, 947-957 (2008).

31. Buonocore, S. et al. Innate lymphoid cells drive interleukin-23-dependent innate intestinal pathology. Nature 464, 1371-1375 (2010).

32. Grusby, M.J. et al. Mice lacking major histocompatibility complex class I and class II molecules. Proc. Natl. Acad. Sci. USA 90, 3913-3917 (1993).

33. Grusby, M.J., Johnson, R.S., Papaioannou, V.E. \& Glimcher, L.H. Depletion of CD4 $+\mathrm{T}$ cells in major histocompatibility complex class II-deficient mice. Science 253, 1417-1420 (1991).

34. Bettelli, E. et al. Reciprocal developmental pathways for the generation of pathogenic effector TH17 and regulatory T cells. Nature 441, 235-238 (2006).

35. Allenspach, E.J., Lemos, M.P., Porrett, P.M., Turka, L.A. \& Laufer, T.M. Migratory and lymphoid-resident dendritic cells cooperate to efficiently prime naive CD4 T cells. Immunity 29, 795-806 (2008).

36. Hill, D.A. et al. Metagenomic analyses reveal antibiotic-induced temporal and spatial changes in intestinal microbiota with associated alterations in immune cell homeostasis. Mucosal Immunol. 3, 148-158 (2010).

37. Salzman, N.H. et al. Enteric defensins are essential regulators of intestinal microbial ecology. Nat. Immunol 11, 76-83 (2010).

38. Bry, L., Brigl, M. \& Brenner, M.B. CD4+-T-cell effector functions and costimulatory requirements essential for surviving mucosal infection with Citrobacter rodentium. Infect. Immun. 74, 673-681 (2006).

39. Margulies, M. et al. Genome sequencing in microfabricated high-density picolitre reactors. Nature 437, 376-380 (2005).

40. McKenna, P. et al. The macaque gut microbiome in health, lentiviral infection, and chronic enterocolitis. PLoS Pathog 4, e20 (2008).

41. Caporaso, J.G. et al. QIIME allows analysis of high-throughput community sequencing data. Nat. Methods 7, 335-336 (2010).

42. Lozupone, C. \& Knight, R. UniFrac: a new phylogenetic method for comparing microbial communities. Appl. Environ. Microbiol. 71, 8228-8235 (2005).

43. Lozupone, C.A., Hamady, M., Kelley, S.T. \& Knight, R. Quantitative and qualitative $\beta$ diversity measures lead to different insights into factors that structure microbial communities. Appl. Environ. Microbiol. 73, 1576-1585 (2007).

44. Hand, T.W. et al. Acute gastrointestinal infection induces long-lived microbiota-specific T cell responses. Science 337, 1553-1556 (2012).

45. Schindelin, J. et al. Fiji: an open-source platform for biological-image analysis. Nat. Methods 9, 676-682 (2012). 